\title{
Salvage Total Pelvic Exenteration with Bilateral V-Y Advancement Flap Reconstruction for Locally Recurrent Rectal Cancer
}

\author{
Jo Tashiro Shigeki Yamaguchi Toshimasa Ishii Hirokazu Suwa \\ Hiroka Kondo Asami Suzuki Mitsuo Miyazawa Isamu Koyama \\ Department of Gastroenterological Surgery, Saitama Medical University International \\ Medical Center, Hidaka, Japan
}

\section{Key Words}

$\mathrm{V}$-Y advancement flap $\cdot$ Total pelvic exenteration · Recurrent rectal cancer

\begin{abstract}
Total pelvic exenteration for locally recurrent rectal cancer typically requires extensive excision of the pelvic floor with perineal skin. Due to the extensiveness of the procedure and its non-curative nature, it is controversial as purely palliative therapy. A 66-year-old male patient who had undergone abdominoperineal resection at another hospital 8 years prior was admitted to our hospital. During radiation and chemotherapy for 2 years, he complained of perineal pain, discharge, cacosmia and bleeding from a recurrent tumor. The $10 \times 8 \mathrm{~cm}$ recurrent tumor was exposed on the perineum and the patient suffered from serious discomfort in his daily life during walking or sitting. We performed total pelvic exenteration with partial sacrectomy, after which the large perineal defect was reconstructed with a bilateral V-Y gluteus maximus advancement flap in approximately $120 \mathrm{~min}$. The patient's postoperative course was satisfactory and his quality of life markedly improved.
\end{abstract}

\section{Introduction}

The acceptability of palliative surgery for patients with locally recurrent rectal cancer has been the topic of previous discussions. The curative rate of resection for locally recurrent rectal cancer is reported as $30-50 \%$, and a higher recurrence rate of $66 \%$ has been reported after total pelvic exenteration (TPE) for locally recurrent rectal cancer [1]. Al-

Jo Tashiro, MD

Department of Gastroenterological Surgery

Saitama Medical University International Medical Center

Yamane, Hidaka-shi, Saitama 350-1298 (Japan)

E-Mail tashiro@saitama-med.ac.jp 
Tashiro et al.: Salvage Total Pelvic Exenteration with Bilateral V-Y Advancement Flap Reconstruction for Locally Recurrent Rectal Cancer

though survival time benefits are uncertain, there have been improvements in patients' quality of life following resection for locally recurrent rectal cancer. However, this strategy remains controversial because it entails extensive non-curative invasive surgery. In general, palliative surgery should be safe and provide fewer complications for patients with a limited life span. Salvage TPE is extremely invasive, but in some cases it is the only procedure available to alleviate serious clinical conditions of pain, discharge, cacosmia and bleeding. However, after the procedure, perineal wound complications can prolong postoperative hospital stay because of the large pelvic dead space and perineal skin defect. Therefore, it is necessary to perform reconstruction using the flap technique with the rectus abdominis, gracilis or gluteus maximus muscles. In the present case, salvage TPE was performed with a $\mathrm{V}$-Y bilateral gluteus maximus advancement flap. The patient's postoperative course was satisfactory and his quality of life markedly improved.

\section{Case Report}

A 66-year-old male patient who had undergone abdominoperineal resection at another hospital 8 years prior was admitted to our hospital. No significant family history was found. Adjuvant radiotherapy to the whole pelvis was recommended for high-risk stage II cancer, T4b (prostate), N0, and M0 according to the TNM classification. After a diagnosis of local recurrence, chemotherapy and additional radiotherapy for the exposed recurrent rectal tumor were performed but were ineffective. For 2 years, the patient complained of persistent perineal pain, discharge, cacosmia and bleeding from the recurrent tumor.

Complete blood count and biochemical tests were almost normal except for tumor markers of carcinoembryonic antigen at $118.3 \mathrm{mg} / \mathrm{dl}$ and cancer antigen $19-9$ at $59.3 \mathrm{mg} / \mathrm{dl}$. The patient's general condition was good; however, a $10 \times 8 \mathrm{~cm}$ recurrent tumor exposed on the perineal midline produced serious discomfort during daily life when he was either walking or sitting. A narcotic analgesic was administered to address the pain (fig. 1a). Computed tomography and magnetic resonance imaging showed a $10-\mathrm{cm}$ bulky mass in the perineum with complicated phlegmonous inflammation. Recurrent cancer had infiltrated into the prostate, the sacral bone (S4) and the gluteus maximus muscle. A solitary lung metastasis was also detected.

TPE with partial sacrectomy was performed. Although the resection was R1 with an insufficient surgical margin, there was no macroscopic residual tumor. The large perineal defect was reconstructed with a bilateral $\mathrm{V}-\mathrm{Y}$ advancement flap using the gluteus maximus muscle. Total surgical time and blood loss were $561 \mathrm{~min}$ and $870 \mathrm{~g}$, respectively. With the patient in the prone jack-knife position, the perineal portion was resected after mobilization of the abdominal segment. The tumor and tissue damaged by irradiation was resected during partial sacrectomy (fig. 1b). The extensive perineal resection with sacrectomy caused a large skin defect which was reconstructed with a bilateral V-Y flap using the gluteus maximus fasciocutaneous tissue.

Reconstruction of the perineum was completed with the patient in the prone position. A bilateral V-shaped incision was made in the skin (fig. 2a). The subcutaneous tissue and the gluteus maximus fascia were divided for the entire portion of the skin incision. Both the superior and the inferior gluteal arteries were preserved. The bilateral flaps were advanced toward the midline and sutured in the following three layers: the muscle, the fasciosubcutaneous tissue and the skin (fig. 2b). The large defect was closed using a V-Y-shaped reconstruction without tension (fig. 3a). The reconstruction surgical time was approximately $120 \mathrm{~min}$. In the supine position, pedicled omentoplasty was patched in the pelvic region to 
Tashiro et al.: Salvage Total Pelvic Exenteration with Bilateral V-Y Advancement Flap Reconstruction for Locally Recurrent Rectal Cancer

decrease the dead space, and a drain was placed in the pelvic floor. Finally, colostomy and ileal conduit were created through the rectus muscle.

Postoperatively, the patient remained in a supine position for a week to avoid pressure and stretch on the flap. On day 3, the hip joint permitted flexure to $30 \%$ in bed. From day 7 , standing position was allowed, and from day 14, the patient was allowed to sit. He was gradually able to resume walking and was discharged on postoperative day 20 . There were no late complications such as pelvic collection, perineal wound dehiscence or lower limb weakness after 1 month (fig. 3b). Moreover, the patient was able to return to normal daily life without serious complaints. His long-term functional outcome, such as walking and sitting, was sufficiently satisfactory. According to pathological findings, the recurrent tubular adenocarcinoma was infiltrating into the prostate and surrounding tissues, with a positive circumferential margin. Fortunately, there has been no evidence of local recurrence, and the solitary lung metastasis has remained stable for 2 years.

\section{Discussion}

Although controversial, palliative surgery for locally recurrent rectal cancer is an effective treatment. The local recurrence rate after rectal cancer resection has been reported to be anywhere from 7 to $33 \%$ [1]. The high recurrence rate is considered to be related to short surgical margins during primary resections. Local recurrence often causes serious clinical conditions of pain, discharge, cacosmia and bleeding. Although most patients receive palliative radiotherapy, its effect is limited and difficult living conditions persist. In such situations, salvage TPE provides the possibility of improving quality of life. This procedure is extremely invasive and technically difficult because the bulky tumor easily involves other organs and there is a limited anatomical plane. In addition, postoperative complications such as fibrosis and edema generally increase after radiotherapy or complicated procedures such as urinary tract reconstruction. The curative resection rate of locally recurrent rectal cancer has been reported as 30-50\%. However, a high recurrence rate of $66 \%$ after TPE for locally recurrent rectal cancer has also been reported [1]. Although previous studies have concluded that both complication and recurrence rates are high after TPE for recurrent cancer, only resection provides the possibility of cure or at least an improvement in quality of life. While it is important to prevent local recurrence when determining the extent of skin resection, it is also important to minimize the skin defect as much as possible. Although a quick intraoperative pathological diagnosis is difficult because of strong fibrosis, the first priority in cases of resection should be to secure oncological safety.

A serious problem, which can prolong the postoperative hospital stay after TPE, is perineal wound complications. Extensive resection for a recurrent bulky tumor produces a large pelvic dead space and perineal skin defect; therefore, it is necessary to perform flap reconstruction using the rectus abdominis, gracilis or gluteus maximus muscles (table 1). Use of such myocutaneous flaps has been reported to reduce wound dehiscence and infection $[2,3]$.

Use of the rectus abdominis myocutaneous flap for perineal defects was first described in 1984 [4]. This flap, supplied by the inferior epigastric artery, reaches any defect of approximately $25 \mathrm{~cm}$ from the groin such as those of the perineum, sacrum and vagina [5]. The rectus abdominis flap, which is voluminous and well-vascularized, is easily accessed through a midline incision. Perineal wound complications after reconstruction using the rectus abdominis myocutaneous flap have been reported as being from 0 to $30 \%$ [6]. In addition, 
Tashiro et al.: Salvage Total Pelvic Exenteration with Bilateral V-Y Advancement Flap Reconstruction for Locally Recurrent Rectal Cancer

preoperative chemoradiation has been reported as a negative factor for perineal wound complications.

The gracilis muscle flap, supplied by the medial circumflex femoral artery, is located 10$14 \mathrm{~cm}$ from the pubic tubercle. Recently, reports using the gracilis muscle flap to repair perineal defects have described a decrease in pelvic abscesses from 46 to $12 \%$. The primary wound healing rate also significantly improved from 33 to 63\% [7]. Another advantage of the gracilis flap is that the abdominal wall is not involved, therefore there is no need to create a colostomy or ileal conduit. This flap is particularly useful for small perineal defects and easily reaches the distal portions of the pelvis. Disadvantages of the gracilis flap are that it is a relatively small tissue and is inadequate for large defects and that the blood supply is unstable, which can lead to flap ischemia [8].

The gluteus flap is supplied by internal iliac artery branches, the superior and inferior gluteal arteries, which also communicate with the external iliac artery branches, the deep femoral artery and the medial circumflex femoral artery. This flap was initially reported for perineal defects by Shaw and Futrell in 1978 [9]. Scheflan et al. [10] reported the V-Y advancement flap using the gluteus maximus muscle for treatment of decubitus ulcers in the sacral and ischial regions with 33.3\% perineal complications. Holm et al. [11] reported using a bilateral gluteal flap to close a large perineal defect with four layers of suture and specific postoperative rehabilitation planning.

Palliative resection for recurrent rectal cancer with a V-Y flap was reported by Goi et al. [12]. The patient survived for more than 1 year without local recurrence and perineal pain. The bilateral V-Y flap was proven as a safe and effective method after resection of a large perineal tumor, even though it was a case of recurrent rectal cancer.

In the present case, both the internal iliac and the gluteal arteries were totally preserved. A large specimen with the sacral bone was removed with the patient in the prone jack-knife position. There was a large defect in the perineum, and a double stoma was necessary at the abdominal wall. In the present case, a bilateral V-Y gluteus maximus flap was the most reasonable method to complete this procedure because the rectus abdominis flap was unsuitable for creating a double stoma, and the gracilis flap was too small to reconstruct the large defect.

Holm et al. [11] reported that one or two plastic surgeons usually perform reconstruction with myocutaneous flaps. While it may be possible for trained colorectal surgeons to reconstruct perineal defects, generally an experienced plastic surgeon should be included in the surgical team for flap reconstruction.

In conclusion, although palliative TPE is an invasive surgery with non-curative intent, it is the only method currently available to resolve serious complaints associated with recurrent rectal cancer. Reconstruction using a V-Y flap for large perineal defects is safe and feasible even in palliative surgery situations.

\section{Disclosure Statement}

The authors have no conflict of interest. This study received no grant support or other assistance. 
Tashiro et al.: Salvage Total Pelvic Exenteration with Bilateral V-Y Advancement Flap Reconstruction for Locally Recurrent Rectal Cancer

\section{References}

1 Avradopoulos KA, Vezeridis MP, Wanebo HJ: Pelvic exenteration for recurrent rectal cancer. Adv Surg 1996;29:215-233.

-2 Ando H, Ito K, Torii S, Kasai Y, Akiyama S, Nakao A: Pedicle myocutaneous flaps for reconstruction following total pelvic exenteration of intrapelvic recurrent rectal cancer: report of a case. Surg Today 2001;31:363366.

3 Nisar PJ, Scott HJ: Myocutaneous flap reconstruction of the pelvis after abdominoperineal excision. Colorectal Dis 2009;11:806-816.

$\checkmark 4$ Shukla HS, Hughes LE: The rectus abdominis flap for perineal wounds. Ann R Coll Surg Engl 1984;66:337339.

5 Wiatrek RL, Thomas JS, Papaconstantinou HT: Perineal wound complications after abdominoperineal resection. Clin Colon Rectal Surg 2008;21:76-85.

6 Tei TM, Stolzeburg T, Buntzen S, Laurberg S, Kjeldsen H: Use of transpelvic rectus abdominis musculocutaneous flap for anal cancer salvage surgery. Br J Surg 2003;90:575-580.

7 Shibata D, Hyland W, Busse P: Immediate reconstruction of the perineal wound with gracilis muscle flaps following abdominoperineal resection and intraoperative radiation therapy for recurrent carcinoma of the rectum. Ann Surg Oncol 1999;6:33-37.

8 Di Mauro D, D’Hoore A, Penninckx F, De Wever I, Vergote I, Hierner R: V-Y bilateral gluteus maximus myocutaneous advancement flap in the reconstruction of large perineal defects after resection of pelvic malignancies. Colorectal Dis 2009;11:508-512.

-9 Shaw A, Futrell JW: Cure of chronic perineal sinus with gluteus maximus flap. Surg Gynecol Obstet 1978;147:417-420.

$\longrightarrow 10$ Scheflan M, Nahai F, Bostwick J: Gluteus maximus island musculocutaneous flap for closure of sacral and ischial ulcers. Plast Reconstr Surg 1981;68:533-542.

11 Holm T, Ljung A, Haggmark T, Jurell G, Lagergren J: Extended abdominoperineal resection with gluteus maximus flap reconstruction of the pelvic floor for rectal cancer. Br J Surg 2007;94:232-238.

12 Goi T, Koneri K, Katayama K, Hirose K, Takashima O, Mizutani Y, Baba H, Yamaguchi A: Modified gluteus maximus V-Y advancement flap for reconstruction of perineal defects after resection of intrapelvic recurrent rectal cancer: report of a case. Surg Today 2003;33:626-629.

Table 1. Characteristics of different flaps

\begin{tabular}{llll}
\hline Characteristic & Flap & & \\
\cline { 2 - 4 } & V-Y gluteus flap & rectus abdominis flap & gracilis flap \\
\hline Type of flap & advancement & transfer rotate & transfer rotate \\
Patient position & prone & lithotomy & lithotomy \\
Pedicle of feeding artery & inferior gluteal artery & inferior epigastric artery & profunda femoris artery \\
Contraindication & resection of total internal & multiple stomas/previous & large defect \\
& iliac artery & abdominal surgery & \\
Large defect & $\circ$ & $\circ$ & $\times$ \\
Vaginal reconstruction & $\circ$ & $\circ$ & $\circ$ \\
Stoma creation & $\circ$ & $\times$ & $\circ$ \\
Technical difficulty & $\times$ & $\circ$ & $\circ$ \\
Wound healing rate & $86-100 \%$ & $54-100 \%$ & $63-100 \%$ \\
\hline
\end{tabular}

$\circ=$ Advantage $x=$ disadvantage . 


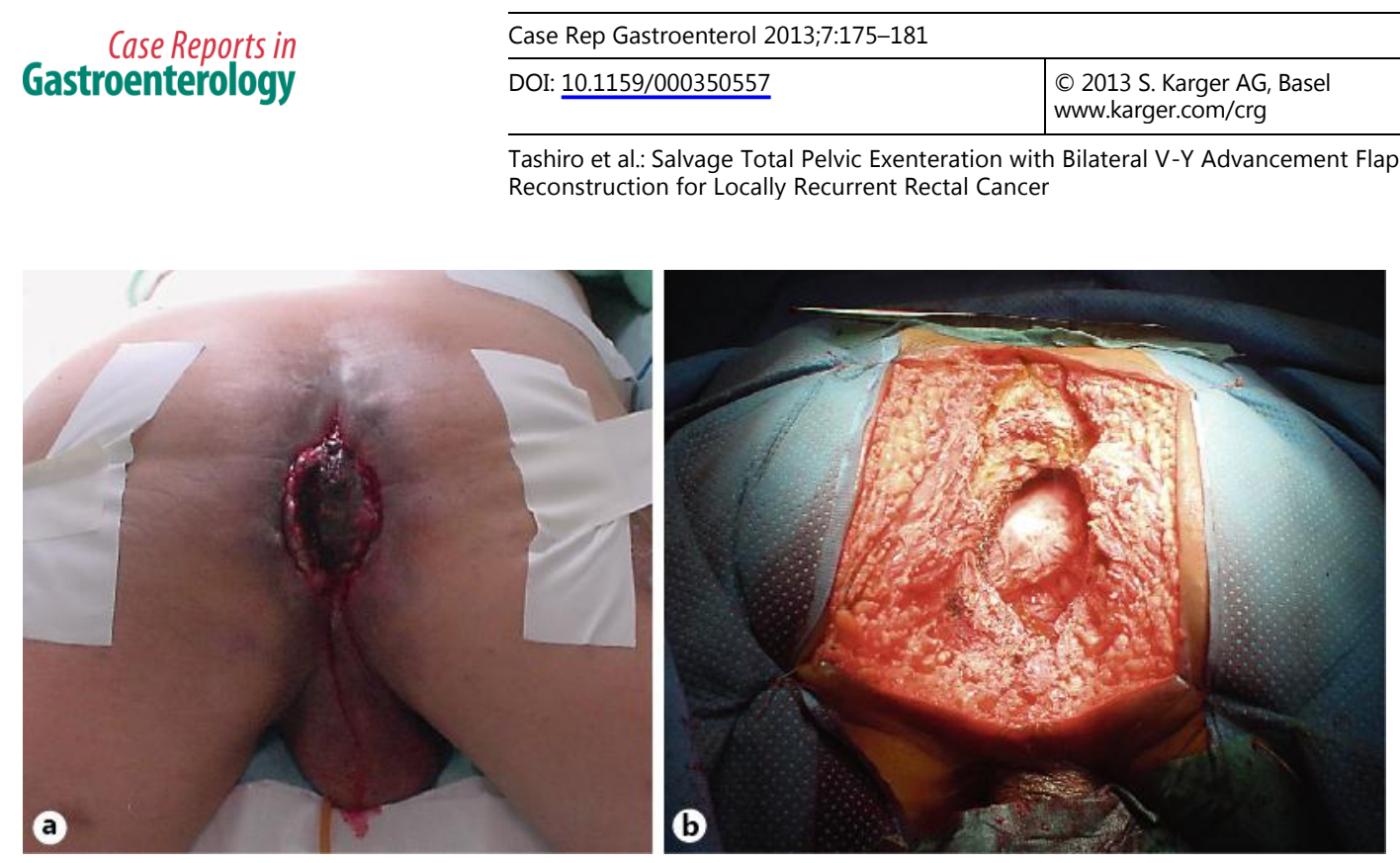

Fig. 1. a The $10 \times 8 \mathrm{~cm}$ tumor was exposed on the suture line, surrounded by complicated phlegmonous inflammation. $\mathbf{b}$ The large perineal defect after TPE with partial sacrectomy.
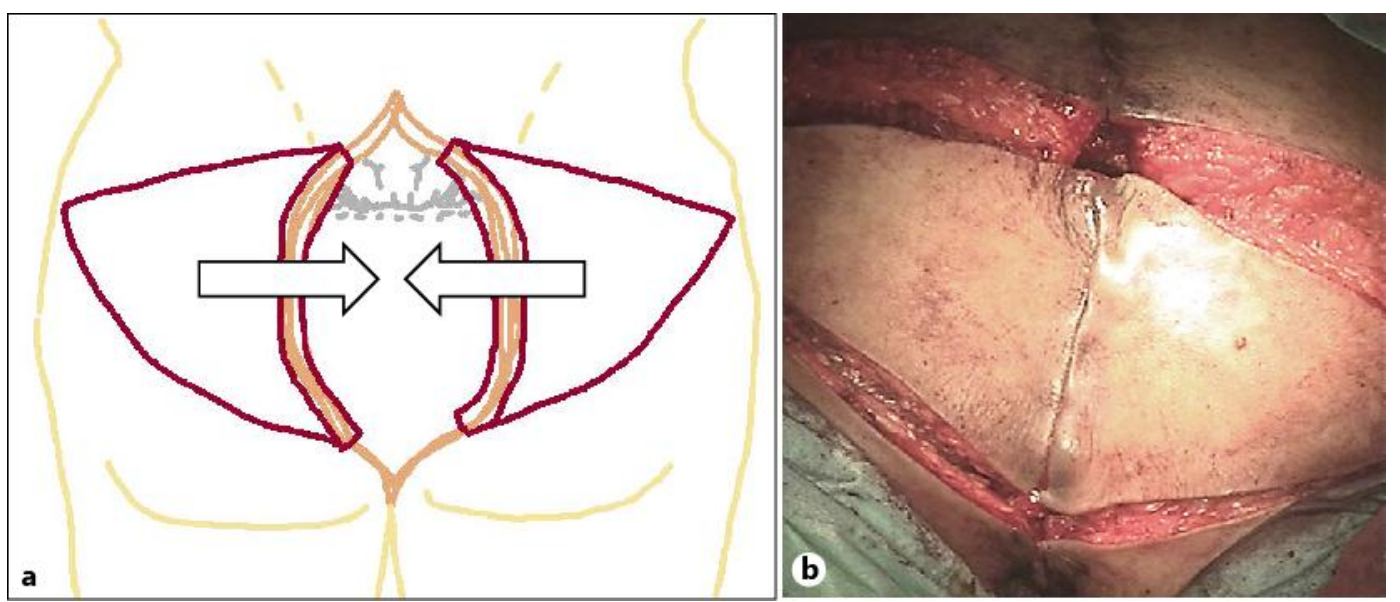

Fig. 2. a Design of the V-shaped skin incision for the V-Y advancement flap. b Both flaps were advanced toward the midline. 
Tashiro et al.: Salvage Total Pelvic Exenteration with Bilateral V-Y Advancement Flap Reconstruction for Locally Recurrent Rectal Cancer
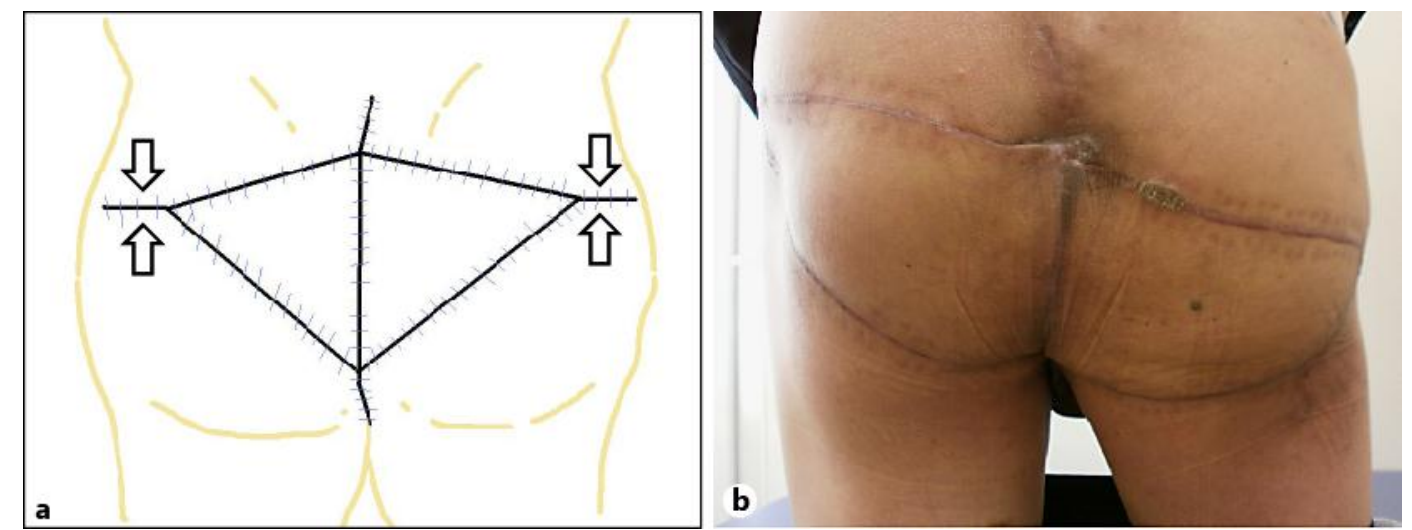

Fig. 3. a Completion of the V-Y-shaped reconstruction. b Findings 4 months after surgery. 